\title{
Measuring shared knowledge with group false memory
}

Research has shown that when
we make decisions, we are
influenced by biases relating
to the way we structure
knowledge in our brains. If these
biases are shared by others, they
can be exaggerated. This often
results in false memories, which
is the recall or recognition of
phenomena that did not occur.
Yoshiko Arima and colleagues
at Kyoto University of Advanced
Science investigated the
conditions that could lead to
false memories within a group
process, and the relationship
between false memories and
shared knowledge structure.
ollaborative groups are known
to create a higher level of memon
accuracy than individuals. This is thought to be the result of a larger to revation pool, the opportunity making Despite their improcilon. collaborative groups tend to make errors in recall tasks. Several studies have found that people are more confident about the accuracy of memories recalled by a group than those recalled by individuals, even when these memories are false. Taken together, this suggests that group false memory occurs when all group members fail to detect errors.

\section{REACHING CONSENSUS} IN GROUP DECISIONS There is wide variability in the time taken to reach consensus when making a decision through a group influence the likelihood of reaching agreement are within-group discrepancy (differences among group members) and between-group discrepancy (difference among groups). The form ins been considered the basis for for intergroup disagreements. However previous research has suggested that these behaviours can influence each other. Studies have found that between-group discrepancies influence not only intergroup processes but also intragroup processes.

\section{FACTORS INFLUENCING} GROUP CONSENSUS

Group polarization is the tendency for a group to make decisions that are more extreme than the initial inclination of its members (known as the mean tendency). Groups polarize

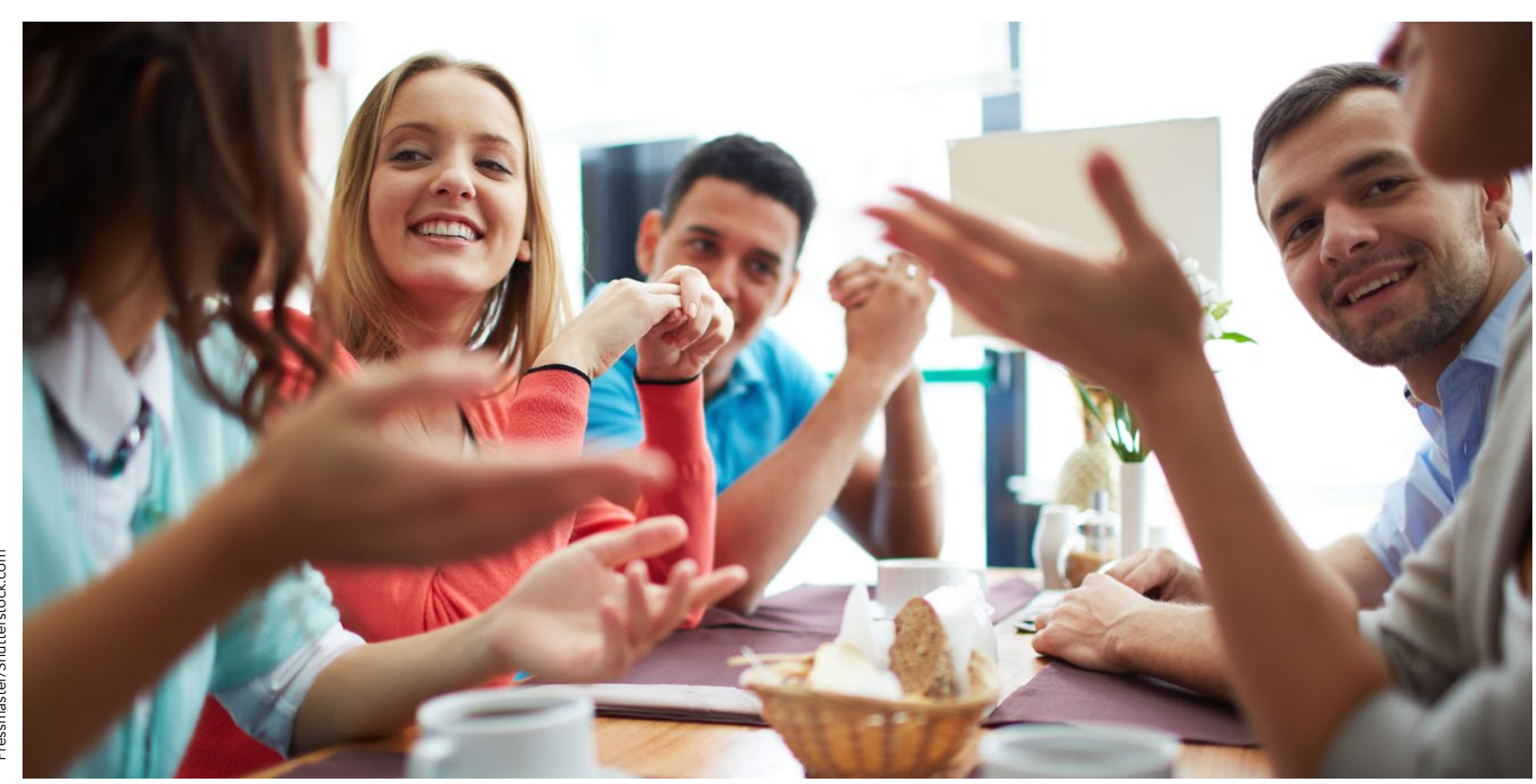

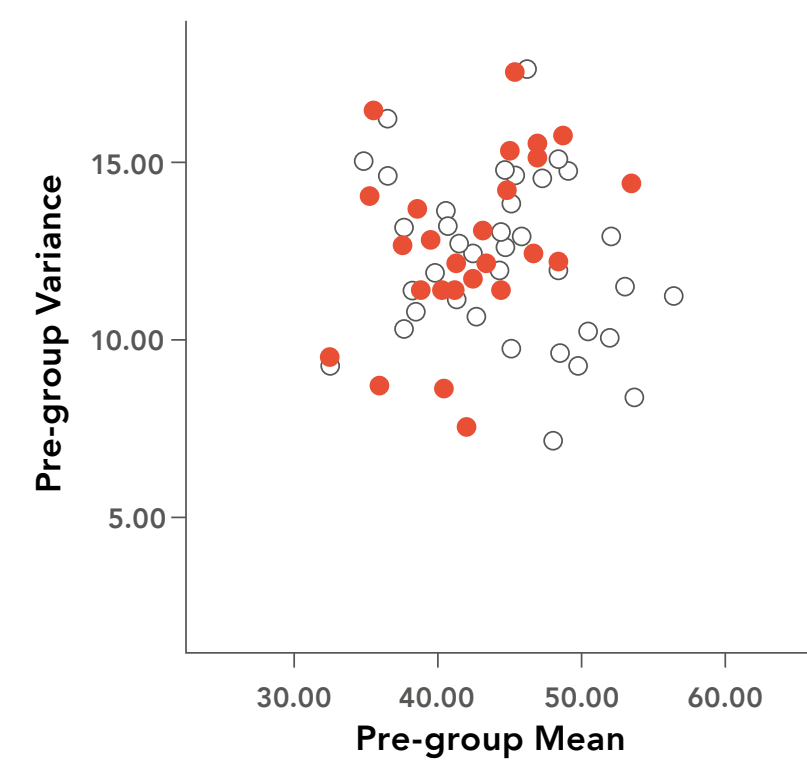

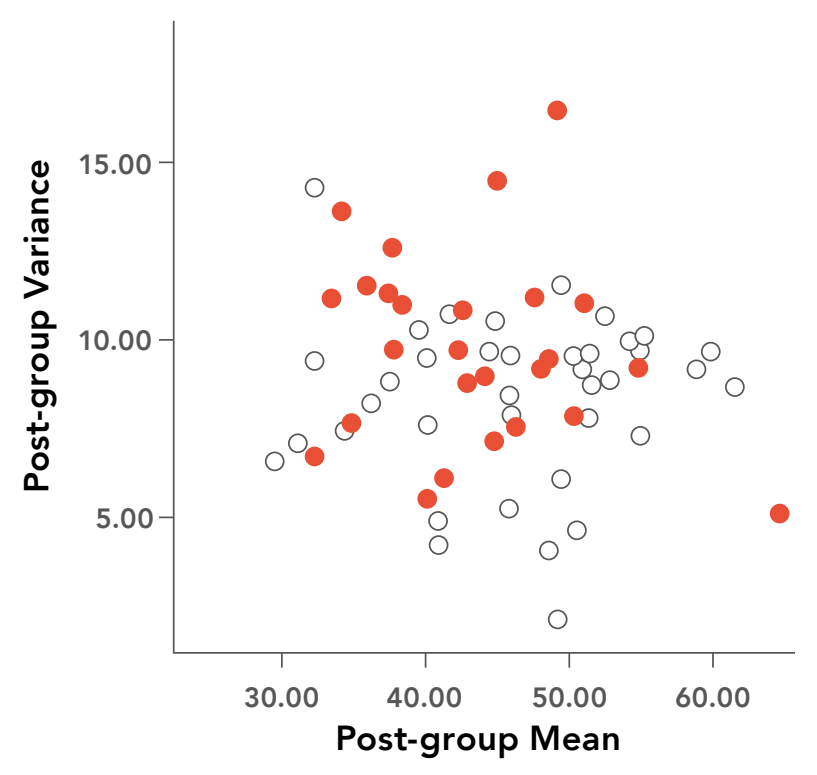

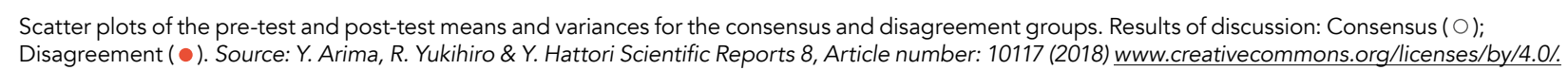

tendency of whole sample even though they do not know which direction it was. Using the group polarization paradigm. research conducted by Dr Yoshiko Arima based at Kyoto University of Advanced Science, explored the conditions that lead to intragroup disagreement and attitude change following a disagreement among 269 participants. Her results show that the probability of consensus was low when the group means differed from the mean of the whole sample. When small differences among group members were found, depolarization (reverse direction to the polarization) followed disagreement. This suggests that the groups which deviated most from the to cause within-group disagreement while within-group differences determined the direction of attitude change following disagreement within the group.

Groups seem to reach a consensus or not randomly, however, they are controlled by the variance of the whole society. There is an 'Invisible Hand' who controls public opinion from one pole to another. The basic assumption of Dr Arima's study is the invisible han of our shared knowledge structure that needs identity and difference. The problem was, how to measure it?

Dr Arima investigated the effect of shared knowledge, manipulated using associated or randomly ordered word lists, on the association between group remembering and group polarisation. In one experiment, 159 university students answered a questionnaire about the common stereotype that blood type determines personality. Half were given lists of words that were consistent with this idea and the oth ordered word lists. After completing the question aire, students were tested on how many words they could recall questions the hapeared in the in either a group or an individual setting. The results demonstrated that reduced the groups' abilthe word list incorrect answers, compared with the individual condition.

In another experiment, 131 high school and university students were divided into three groups: group members having the same blood type (low-diversity). members with two different blood types (medium-diversity), and members with (high-diversity). This condition induces three levels of variety of memories tests, participants take a recognition test that includes the presented associative

There is wide variability in the time taken to reach consensus when making a decision in a group context. 


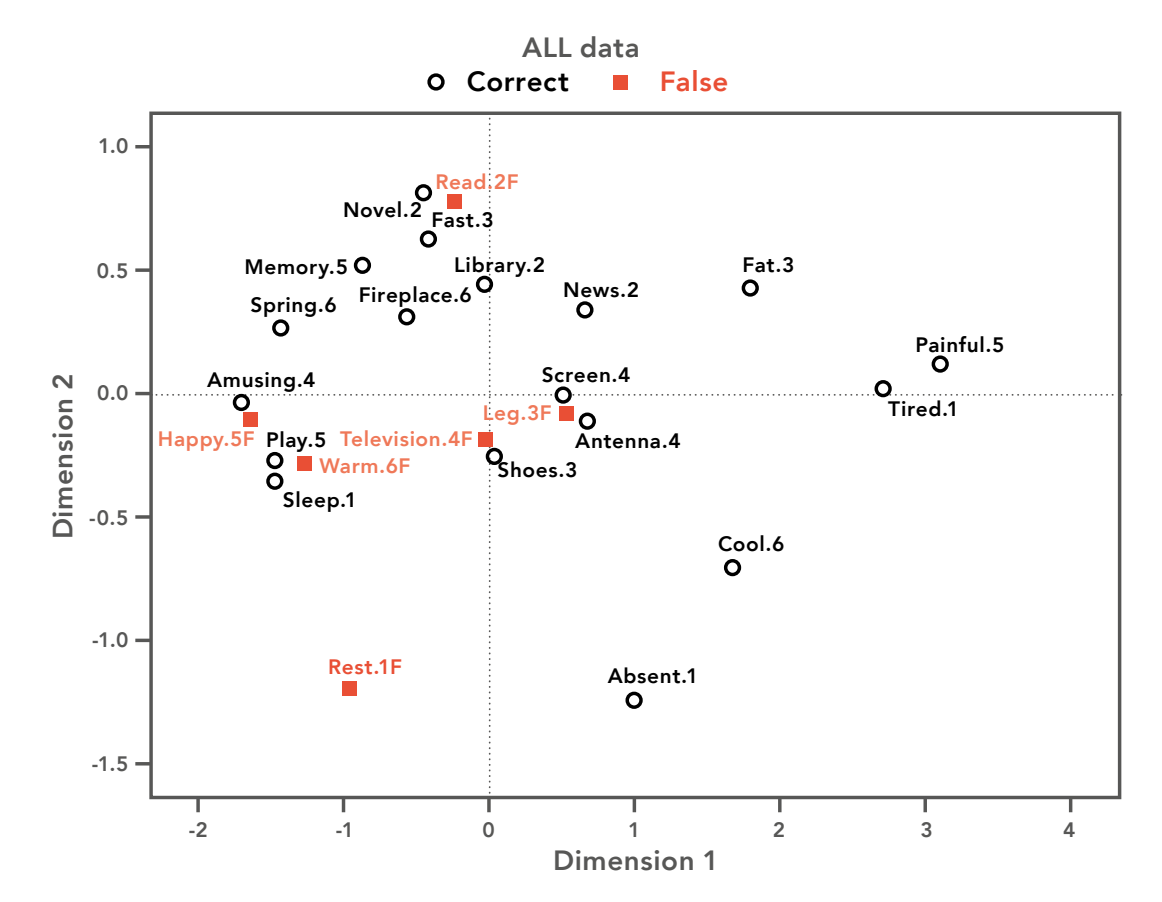

words (known as targets), non-presented consistent word list condition than for associative words (critical words whereby $\quad$ the randomised word list condition, and
a response indicates a false memory)
secondly, that after collaboration, group and non-presented irrelevant words (known as distractors whereby a response indicates an error). Usually, the proportion of false memory is much higher than that of errors.

The activation-monitoring hypothesis predicts that false memory occurs when critical words continually become activated, eventually producing a memory. Evidence for this idea has been obtained from studies showing that false recognition increases, and accurate recognition decrease with the number of targets in a word list. hypothesis, Dr Arim predicted that false memory would be dependenton the strength of the critical word as a semantic cue at the moment of learning 'Knowledge structure' represents the associative-semantic network related to specific cues. 'Shared knowledge structure' is the intersection of group members' knowledge structures. Two hypotheses were tested: firstly, that group false memory would be larger for the secondly, that after collaboration, group errors and the difference between the consistent and randomised word lists at the pre-test stage would remain the same.

Using the DRM, they conducted the first experiment in 121 university students. Participants collaborated to select keywords using between-subject condition from a consistent or randomised word list. Between-subjects means that groups are compared with each other. The proportion of false responses was larger than that centron consistent word list condition and group for both word lists, which was not caused contagion.

A second study on 119 university students used a within-subjects design, where differences within a group are compared and confirmed the findings of the first experiment. False memories increased after group collaboration regardless of the meaning of the words, maintaining the difference between the consistent and

The activation-monitoring hypothesis predicts that false memory will occur when critical words become activated continually, and eventually produce a memory.
ALSCAL results for all data (S-stress $=0.13$, dimension represents the plas . The horizontal pleasure scales. The vertical dimension seems
to discriminate between intellectual activities Or rest. Source: Arima, Y. Vol 110, Issue 2, 2012.
Copyright 1 [2018] DOI: [10.2466/01.11.17.21. PRo.110.2.607-623].

randomised word lists. Dr Arima also mense latency of each demonstrated that there are reationships between response latency and the score of knowledge structure analysed by MultDimensional Scale.

\section{CONCLUSIONS}

DrArima's findings demonstrate that group false memory is shaped by a shared knowledge structure, a process which can have both positive and negative consequences. With shared knowledge, it is easy to exchange concepts, but this may mean that increasing mistakes vesulting to group polarization. If a group group members the sroup will among to detect these mistakes. However, with the group process, there will be difficulties as a result of miscommunication. To

overcome this problem, it is necessa

to have a complex shared knowledge structure. However, a compromise may exist between shared knowledge and the amount of shared memory.

The results of the current study show that group false memory can be an index for a pre-stored and a post-shared knowledge structure. False memories in consistent word lists can be used as a measure to understand what associations are shared in pre-stored can revea, and randomised word lists creates a poststion wh which a group

\section{Research Objectives}

Dr Arima's research investigates shared knowledge structure with the aim of estimating complexities of our elligence.

\section{Detail}

\section{DrYoshiko Arima}

Department of Psychology

18Yamanch

Ukyo-ku, Kyoto 615-8577, Japan

\section{Bio}

Dr Yoshiko Arima obtained her undergraduate degree within the Faculty of Human Science, Osaka University in 1980 before obtaining her PhD in Social Physiology. She is currently Professor at the Department of Psychology. Faculty of Humanities, Kyoto University of Advanced Science.

\section{Collaborators}

- Roji Yukihiro

- Yosuke Hattori

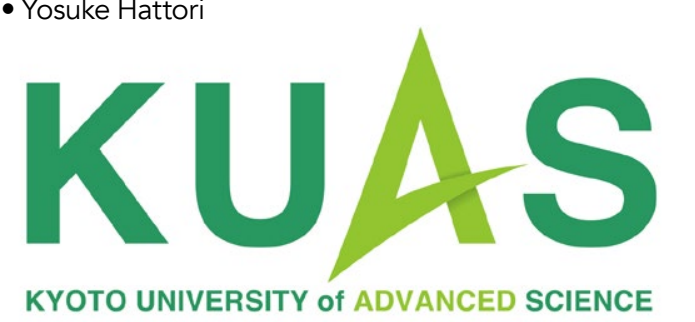

KYOTO UNIVESTY OT ADVANCED SCIENCE

\section{References}

Arima, Y. (2012). Effect of Group Means on the Probability ofConsensus. Psychological Reports, 110(2), 607-623.

Arima, Y. (2013). Effect of word-list consistency on the correlation between group memory and group

Arima, Y., Yukihiro, R., \& Hattori, Y. (2018). Measuring shared knowledge with group false memory. Scientific Reports, 8(1) 10117

\section{Personal Response}

\section{What is the focus of your future research?}

III The focus of my future research is to integrate group process studies with collective intelligence studies. Even if
crowd deviated from the truth, expert crowds, or machine crowd deviated from the truth, expert crowds, or machir
learning from expert's data, will perform over the best learning from expent's data, will perform over the best than a person does. However, there is no guarantee that we can understand the complexities in the answer of collective intelligence. The common knowledge effect and cyber cascade polarize crowds and machines may learn the biases. The study on measuring shared know may learn the to prevent polarization of our society.
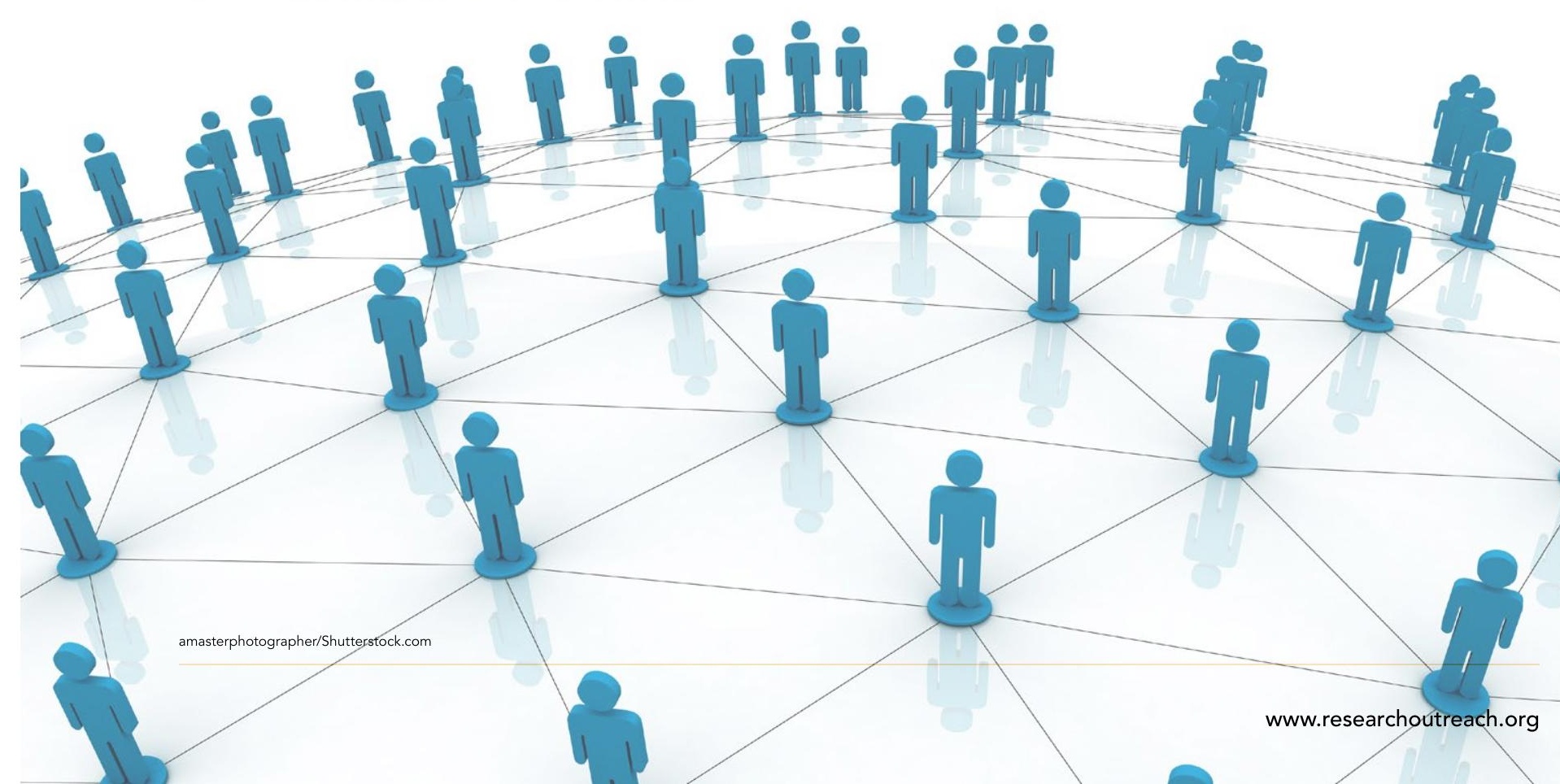